\title{
Domination of the rectangular queen's graph
}

\author{
Sándor Bozóki \\ Institute for Computer Science and \\ Control (SZTAKI), Budapest, Hungary; \\ Corvinus University of Budapest \\ bozoki.sandor@sztaki.hu \\ István Marosi \\ Kofax-Recognita Zrt., Budapest, Hungary \\ imarosi@gmail.com \\ Péter Gál \\ Kofax-Recognita Zrt., Budapest, Hungary \\ galpetya@gmail.com \\ William D. Weakley \\ Purdue University Fort Wayne, USA \\ weakley@pfw.edu
}

Submitted: Feb 21, 2019; Accepted: Nov 20, 2019; Published: Dec 6, 2019

(C) The authors. Released under the CC BY-ND license (International 4.0).

\begin{abstract}
The queens graph $Q_{m \times n}$ has the squares of the $m \times n$ chessboard as its vertices; two squares are adjacent if they are in the same row, column, or diagonal of the board. A set $D$ of squares of $Q_{m \times n}$ is a dominating set for $Q_{m \times n}$ if every square of $Q_{m \times n}$ is either in $D$ or adjacent to a square in $D$. The minimum size of a dominating set of $Q_{m \times n}$ is the domination number, denoted by $\gamma\left(Q_{m \times n}\right)$.

Values of $\gamma\left(Q_{m \times n}\right), 4 \leqslant m \leqslant n \leqslant 18$, are given here, in each case with a file of minimum dominating sets (often all of them, up to symmetry) in an online appendix. In these ranges for $m$ and $n$, monotonicity fails once: $\gamma\left(Q_{8 \times 11}\right)=6>$ $5=\gamma\left(Q_{9 \times 11}\right)=\gamma\left(Q_{10 \times 11}\right)=\gamma\left(Q_{11 \times 11}\right)$.

Let $g(m)$ [respectively $g^{*}(m)$ ] be the largest integer such that $m$ queens suffice to dominate the $(m+1) \times g(m)$ board [respectively, to dominate the $(m+1) \times g^{*}(m)$ board with no two queens in a row]. Starting from the elementary bound $g(m) \leqslant$ $3 m$, domination when the board is far from square is investigated. It is shown (Theorem 2 ) that $g(m)=3 m$ can only occur when $m \equiv 0,1,2,3$, or $4(\bmod 9)$, with the online appendix showing that this does occur for $m \leqslant 40, m \neq 3$. Also (Theorem 4$)$, if $m \equiv 5,6$, or $7(\bmod 9)$ then $g^{*}(m) \leqslant 3 m-2$, and if $m \equiv 8(\bmod 9)$ then $g^{*}(m) \leqslant 3 m-4$. It is shown that equality holds in these bounds for $m \leqslant 40$.

Lower bounds on $\gamma\left(Q_{m \times n}\right)$ are given. In particular, if $m \leqslant n$ then $\gamma\left(Q_{m \times n}\right) \geqslant$ $\min \{m,\lceil(m+n-2) / 4\rceil\}$.

Two types of dominating sets (orthodox covers and centrally strong sets) are developed; each type is shown to give good upper bounds of $\gamma\left(Q_{m \times n}\right)$ in several cases.

Three questions are posed: whether monotonicity of $\gamma\left(Q_{m \times n}\right)$ holds (other than from $(m, n)=(8,11)$ to $(9,11))$, whether $\gamma\left(Q_{m \times n}\right)=(m+n-2) / 4$ occurs with
\end{abstract}


$m \leqslant n<3 m+2$ (other than for $(m, n)=(3,3)$ and $(11,11)$ ), and whether the lower bound given above can be improved.

A set of squares is independent if no two of its squares are adjacent. The minimum size of an independent dominating set of $Q_{m \times n}$ is the independent domination number, denoted by $i\left(Q_{m \times n}\right)$. Values of $i\left(Q_{m \times n}\right), 4 \leqslant m \leqslant n \leqslant 18$, are given here, in each case with some minimum dominating sets. In these ranges for $m$ and $n$, monotonicity fails twice: $i\left(Q_{8 \times 11}\right)=6>5=i\left(Q_{9 \times 11}\right)=i\left(Q_{10 \times 11}\right)=i\left(Q_{11 \times 11}\right)$, and $i\left(Q_{11 \times 18}\right)=9>8=i\left(Q_{12 \times 18}\right)$.

Mathematics Subject Classifications: 05C69, 05C99

\section{Introduction}

Let $m$ and $n$ be positive integers. We will identify the $m \times n$ chessboard with a rectangle in the Cartesian plane, having sides parallel to the coordinate axes. We place the board so that the center of every square has integer coordinates, and refer to each square by the coordinates $(x, y)$ of its center. Unless otherwise noted, squares have edge length one, and the board is placed so that the lower left corner has center $(1,1)$; sometimes it is more convenient to use squares of edge length two or to place the board with its center at the origin of the coordinate system. By symmetry it suffices to consider the case $m \leqslant n$, which we will assume throughout: the board has at least as many columns as rows.

The square $(x, y)$ is in column $x$ and row $y$. Columns and rows will be referred to collectively as orthogonals. The difference diagonal (respectively sum diagonal) through square $(x, y)$ is the set of all board squares with centers on the line of slope +1 (respectively $-1)$ through the point $(x, y)$. The value of $y-x$ is the same for each square $(x, y)$ on a difference diagonal, and we will refer to the diagonal by this value. Similarly, the value of $y+x$ is the same for each square on a sum diagonal, and we associate this value to the diagonal. Orthogonals and diagonals are collectively referred to as lines of the board.

The queens graph $Q_{m \times n}$ has the squares of the $m \times n$ chessboard as its vertices; two squares are adjacent if they are in some line of $Q_{m \times n}$. A set $D$ of squares of $Q_{m \times n}$ is a dominating set for $Q_{m \times n}$ if every square of $Q_{m \times n}$ is either in $D$ or adjacent to a square in $D$. The minimum size of a dominating set is the domination number, denoted by $\gamma\left(Q_{m \times n}\right)$. A set of squares is independent if no two squares in the set are adjacent.

Almost all previous work on queen domination has concerned square boards. The problem of finding values of $\gamma\left(Q_{n \times n}\right)$ has interested mathematicians for over 150 years. The first published work is that of De Jaenisch [13] in 1862, who gave minimum dominating sets and minimum independent dominating sets of $Q_{n \times n}$ for $n \leqslant 8$. His work was briefly summarized by Rouse Ball [19] in 1892, who considered several other questions about queen domination. In 1901, W. Ahrens [1, Chapter X] gave minimum dominating sets for $Q_{9 \times 9}$, and in 1902-3, K. von Szily [20,21] gave minimum dominating sets of $Q_{n \times n}$ for $10 \leqslant n \leqslant 13$ and $n=17$. Proof that these sets were minimum had to wait for later work, described below. De Jaenisch, Ahrens, and von Szily also worked extensively to find the number of different minimum dominating sets for each $n$, often giving lists with one set 
from each symmetry class. Many of these results were collected by Ahrens in the 1910 edition [2] of his book, and can also be found in its later editions.

More detail and some examples from recent work on domination of $Q_{n \times n}$ can be found in $[26]$.

The first published work on nonsquare boards of which we are aware is in Watkins [22]: the values $\gamma\left(Q_{5 \times 12}\right)=4$ and $\gamma\left(Q_{6 \times 10}\right)=4$ (see Problem 8.4 on p. 132 and Figure 8.19 on p. 137), found by D. C. Fisher.

Say that two minimum dominating sets of $\gamma\left(Q_{m \times n}\right)$ are equivalent if there is an isometry of the $m \times n$ chessboard that carries one to the other.

We have computed $\gamma\left(Q_{m \times n}\right)$ for rectangular chessboards with $4 \leqslant m \leqslant n \leqslant 18$. Results are given in Table 1 ; for most $m$ and $n$ we give a file of minimum dominating sets with one from every equivalence class, unless the number of equivalence classes is large. The online appendix at https://www. combinatorics.org/ojs/index.php/eljc/ article/view/v26i4p45/HTML includes the computational results. For each set, we describe its symmetry and say whether it can be obtained by one of the constructions in Section 3.

\begin{tabular}{|c||c|c|c|c|c|c|c|c|c|c|c|c|c|c|c|}
\hline$n \backslash m$ & 4 & 5 & 6 & 7 & 8 & 9 & 10 & 11 & 12 & 13 & 14 & 15 & 16 & 17 & 18 \\
\hline \hline 4 & 2 & & & & & & & & & & & & & & \\
\hline 5 & 2 & 3 & & & & & & & & & & & & & \\
\hline 6 & 3 & 3 & 3 & & & & & & & & & & & & \\
\hline 7 & 3 & 3 & 4 & 4 & & & & & & & & & & & \\
\hline 8 & 3 & 4 & 4 & 5 & 5 & & & & & & & & & & \\
\hline 9 & 4 & 4 & 4 & 5 & 5 & 5 & & & & & & & & & \\
\hline 10 & 4 & 4 & 4 & 5 & 5 & 5 & 5 & & & & & & & & \\
\hline 11 & 4 & 4 & 5 & 5 & 6 & 5 & 5 & 5 & & & & & & & \\
\hline 12 & 4 & 4 & 5 & 5 & 6 & 6 & 6 & 6 & 6 & & & & & & \\
\hline 13 & 4 & 5 & 5 & 6 & 6 & 6 & 7 & 7 & 7 & 7 & & & & & \\
\hline 14 & 4 & 5 & 6 & 6 & 6 & 6 & 7 & 7 & 8 & 8 & 8 & & & & \\
\hline 15 & 4 & 5 & 6 & 6 & 6 & 7 & 7 & 7 & 8 & 8 & 8 & 9 & & & \\
\hline 16 & 4 & 5 & 6 & 6 & 7 & 7 & 7 & 8 & 8 & 8 & 9 & 9 & 9 & & \\
\hline 17 & 4 & 5 & 6 & 7 & 7 & 7 & 8 & 8 & 8 & 9 & 9 & 9 & 9 & 9 & \\
\hline 18 & 4 & 5 & 6 & 7 & 7 & 8 & 8 & 8 & 8 & 9 & 9 & 9 & 9 & 9 & 9 \\
\hline
\end{tabular}

Table 1: Values of $\gamma\left(Q_{m \times n}\right), 4 \leqslant m \leqslant n \leqslant 18$ (OEIS A274138)

The computation was done with a backtracking algorithm. The backtrack condition minimizes the number of queens placed. If a solution is found with $k$ queens, then the remaining search space is limited to at most $k-1$ queens. The algorithm places a single queen in a position covering the top left cell and does a recursive call to cover all remaining cells. Some heuristics are used also to find the first solution faster: the first queen is placed in the middle of the board (actually in the closest to middle position attacking the top 


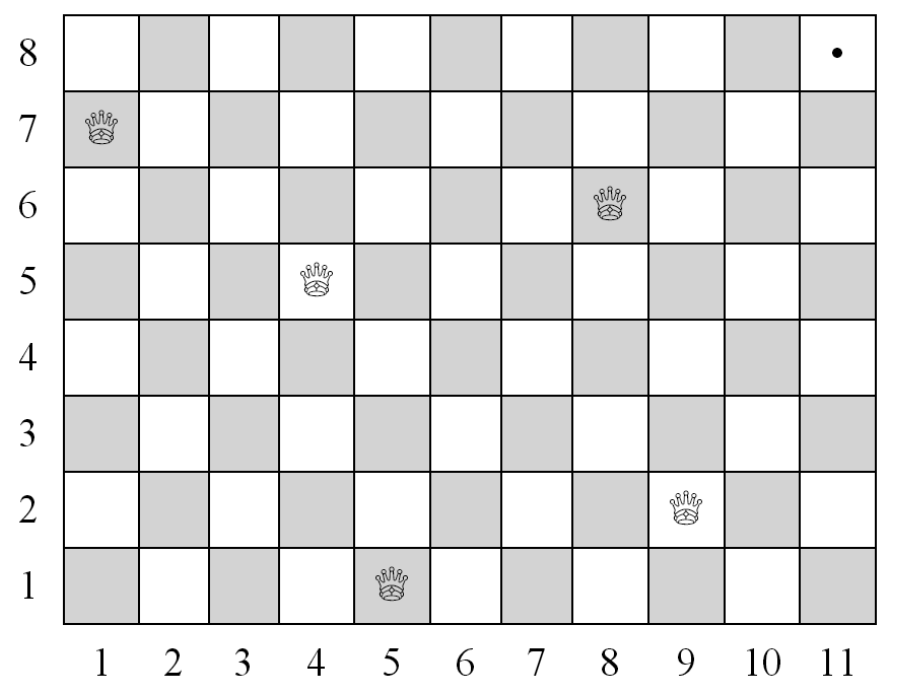

Figure 1: Five queens dominate $Q_{8 \times 11}$ except for one square

left cell); other possible attacking positions are only tried later. Frequently this position is part of a minimal solution.

Once it is shown that there is no solution with $k-1$ queens, a search for other solutions with $k$ queens is made.

Cockayne [7, Problem 1] introduced monotonicity

$$
\gamma\left(Q_{n \times n}\right) \stackrel{?}{\leqslant} \gamma\left(Q_{(n+1) \times(n+1)}\right)
$$

as an open problem (see also in Chartrand, Haynes, Henning and Zhang [6, Conjecture 1.2.1 on page 7 ].

A remarkable observation about $Q_{8 \times 11}$ : six queens (with bold typeface in Table 1) are necessary to dominate it, though five queens are sufficient (and necessary) to dominate each of $Q_{9 \times 11}, Q_{10 \times 11}, Q_{11 \times 11}$. A possible explanation for this is given later. We note that five queens can cover all but one square of $Q_{8 \times 11}$. One of the 8 placements is in Figure 1.

We extend Cockayne's question to the rectangular case.

Question 1. Column-wise monotonicity: Does $\gamma\left(Q_{m \times n}\right) \leqslant \gamma\left(Q_{m \times(n+1)}\right)$ hold for $m \leqslant n$ ? Row-wise monotonicity: Does $\gamma\left(Q_{m \times n}\right) \leqslant \gamma\left(Q_{(m+1) \times n}\right)$ hold for $m \leqslant n,(m, n) \neq(8,11)$ ?

We discuss one type of internal symmetry of minimum dominating sets that frequently occurs. A foursome is a set of four squares $(x+a, y+b),(x-a, y-b),(x-b, y+a)$, $(x+b, y-a)$, where either each of $x, y, a, b$ is an integer or each is half an odd integer, and $a$ and $b$ are unequal and nonzero. The center of the foursome is the point $(x, y)$, which need not be a square center. For examples, see Figure 1 above, the first minimum dominating sets given for $Q_{9 \times 9}$ and $Q_{11 \times 11}$, as well as the first four minimum dominating sets given for $Q_{11 \times 12}$. 


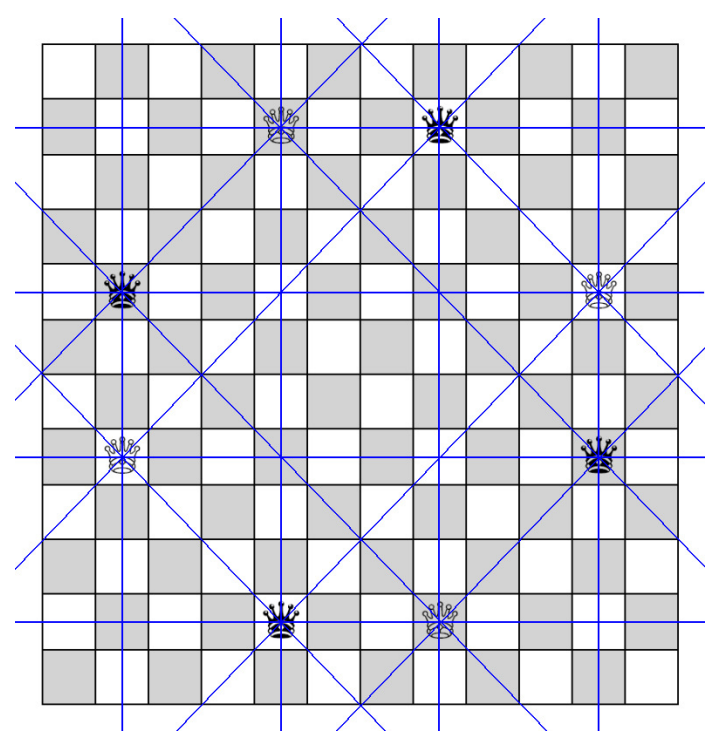

Figure 2: The four squares with white queens are a foursome; the lines through its squares are shown. Reflecting this foursome across any of the four lines through its center gives another foursome (the squares with black queens) that occupies the same lines.

If a foursome $F$ is flipped across any of the four lines through its center, the result is another foursome $F^{\prime}$ that occupies the same lines as $F$; this is illustrated in Figure 2.

Thus if a dominating set $D$ of $Q_{m \times n}$ contains $F$, we may replace $F$ in $D$ with $F^{\prime}$ and obtain a dominating set $D^{\prime}$ of the same size as $D$. Usually $D$ and $D^{\prime}$ are not equivalent.

As an example, we analyze the minimum dominating sets of $Q_{11 \times 17}$, which have size 8. Up to equivalence there are 131 solutions, shown in the file. Of these, 85 have no foursomes, 41 have exactly one foursome, four (\#125, 69, 70, 62) have exactly two foursomes, and one (\#76) has 3 foursomes.

We may define a relation on the set $\mathcal{S}(11,17)$ of minimum dominating sets of $Q_{11 \times 17}$ by saying that two sets are related if either they are equivalent, or flipping a foursome of the first set yields a set equivalent to the second set. This relation is reflexive and symmetric, and its transitive closure gives a partition $\mathcal{P}(11,17)$ of $\mathcal{S}(11,17)$, which may also be regarded as a partition of the set of (isometric) equivalence classes, as we will do.

For example, solution \#125 has two foursomes: one centered at $(12,6)$ with $(a, b)=$ $(4,2)$, and one centered at $(9,7)$ with $(a, b)=(3,-1)$. Flipping the first gives solution $\# 124$. If instead we flip the second, we get the reflection of \#125 across its vertical line of symmetry. This implies that one part of the partition $\mathcal{P}(11,17)$ contains just the equivalence classes of 125 and 124 , and we denote this part by $\{125,124\}$. It is then straightforward to see that $\mathcal{P}(11,17)$ has 85 parts with one member and 20 parts with two members: $\{8,9\},\{20,24\}\{19,23\},\{21,25\},\{22,26\},\{15,27\},\{13,6\},\{12,5\}$, $\{128,129\},\{73,68\},\{127,130\},\{126,131\},\{103,97\},\{125,124\},\{39,44\},\{96,95\}$, $\{63,62\},\{72,70\},\{101,77\},\{100,78\}$. There are also two parts with three members: 
$\{80,71,69\}$ and $\{79,76,75\}$.

It would be possible to reduce the size of the appendix by giving for each $(m, n)$ one solution from each part of the partition $\mathcal{P}(m, n)$ rather than one solution from each isometry equivalence class. But when two solutions differ by the flip of a foursome, it is not clear which is most useful to see, so we have not done this.

\section{Lower bounds on queen domination numbers}

We begin by looking at what happens when the board is far from square.

Proposition 1. If $n \geqslant 3 m-2$, then $\gamma\left(Q_{m \times n}\right)=m$.

Proof. Each queen attacks all squares in her own row, but at most three squares in any other row. Thus $m-1$ queens occupy at most $m-1$ rows and cover at most $3(m-1)$ squares in any row that does not contain a queen. On the other hand, $m$ queens are certainly sufficient.

Note that $\gamma\left(Q_{3 \times 6}\right)=2$ (see the set given immediately after Theorem 2$)$ and $\gamma\left(Q_{5 \times 12}\right)=$ 4 (see the database), but as shown by our computations, for $m=4,6,7, \gamma\left(Q_{m \times n}\right)$ reaches $m$ before $n$ reaches $3 m-2$.

We change viewpoint slightly, focusing on the size of the dominating set rather than the dimensions of the board. For each positive integer $m$, let $g(m)$ be the largest integer such that $m$ queens can cover the $(m+1) \times g(m)$ board. (Proposition 1 asserts $g(m) \leqslant 3 m$.) Let $g^{*}(m)$ be the largest integer such that $m$ queens, no two in a row, can cover the $(m+1) \times g^{*}(m)$ board.

Theorem 2. If $g(m)=3 m$ then $m \equiv 0,1,2,3$, or $4(\bmod 9)$.

Take the board to be a rectangle in the Cartesian plane with sides parallel to the axes, board center at the origin, and squares of edge length two.

Assume that $D$ is a dominating set of size $m$ for the $(m+1) \times 3 m$ board. One of two cases occurs:

1. there is only one empty row, which without loss of generality has index $h, 0 \leqslant h \leqslant m$, and each other row contains exactly one square of $D$, or

2. there are exactly two empty rows; in this case $m$ is even, row 0 contains two squares of $D$, the empty rows are indexed $\pm h$ for some $h, 0<h \leqslant m$, and each other row contains exactly one square of $D$.

In either case:

- if $m \equiv 0$ or $4(\bmod 9)$, then $h \equiv 0(\bmod 3)$;

- if $m \equiv 1$ or $3(\bmod 9)$, then $h \not \equiv 0(\bmod 3)$. 
Proof. The $3 m$ column indices are $x=1-3 m, 3-3 m, \ldots, 3 m-3,3 m-1$ and the $m+1$ row indices are $y=-m, 2-m, \ldots, m-2, m$.

Let $D=\left\{\left(x_{i}, y_{i}\right): 1 \leqslant i \leqslant m\right\}$ be a dominating set of the $(m+1) \times 3 m$-board. Since $|D|=m$ and there are $m+1$ rows, at least one row contains no square of $D$. Let $h$ be the index of such a row. Since $|D|=m$, and a queen covers at most three squares of any line she does not occupy, the $3 m$ squares of row $h$ are each covered exactly once by $D$. For each $(x, y) \in D$, the squares of row $h$ covered by $(x, y)$ are $(x-(y-h), h),(x, h),(x+(y-h), h)$. We can add up the squares of the column indices of the squares in row $h$ in two ways, giving the equation

$$
(1-3 m)^{2}+(3-3 m)^{2}+\ldots+(3 m-1)^{2}=\sum_{i=1}^{m}\left[\left(x_{i}-\left(y_{i}-h\right)\right)^{2}+x_{i}^{2}+\left(x_{i}+\left(y_{i}-h\right)\right)^{2}\right] .
$$

This reduces to

$$
2\left(\begin{array}{c}
3 m+1 \\
3
\end{array}\right)=3 \sum_{i=1}^{m} x_{i}^{2}+2 \sum_{i=1}^{m} y_{i}^{2}-4 h \sum_{i=1}^{m} y_{i}+2 m h^{2} .
$$

For a particular dominating set $D$ we may regard this as a quadratic equation in $h$, so there are at most two empty rows.

If there are two empty rows $h_{1}, h_{2}$, then (1) implies $2 m h_{1}^{2}-4 h_{1} \sum_{i=1}^{m} y_{i}=2 m h_{2}^{2}-$ $4 h_{2} \sum_{i=1}^{m} y_{i}$ and then

$$
\sum_{i=1}^{m} y_{i}=\frac{m\left(h_{1}+h_{2}\right)}{2}
$$

As $|D|=m$, there is exactly one row, say $l$, with two queens, and all rows except $h_{1}, h_{2}, l$ have just one queen. Thus $\sum_{i=1}^{m} y_{i}=-h_{1}-h_{2}+l$. With (2) this implies $-h_{1}-h_{2}+l=m\left(h_{1}+h_{2}\right) / 2$ and then $l=(m+2)\left(h_{1}+h_{2}\right) / 2$. From $-m \leqslant l \leqslant m$ we have $-2 m \leqslant(m+2)\left(h_{1}+h_{2}\right) \leqslant 2 m$ so $-2<h_{1}+h_{2}<2$. But $h_{1}+h_{2}$ is even since all row indices have the same parity. Thus $h_{1}+h_{2}=0$, so $l=0$ and all row indices are even, which implies $m$ is even. So there is $h, 0<h \leqslant m$, such that the empty rows are $\pm h$. Here $\sum_{i=1}^{m} y_{i}=0$ and $\sum_{i=1}^{m} y_{i}^{2}=2\left(\begin{array}{c}m+2 \\ 3\end{array}\right)-2 h^{2}$, and (1) becomes

$$
2\left(\begin{array}{c}
3 m+1 \\
3
\end{array}\right)-4\left(\begin{array}{c}
m+2 \\
3
\end{array}\right)=3 \sum_{i=1}^{m} x_{i}^{2}+2(m-2) h^{2}
$$

If instead there is only one empty row $h$, then we may assume $0 \leqslant h \leqslant m$ by flipping across the $x$-axis if necessary. Then $\sum_{i=1}^{m} y_{i}=-h$ and $\sum_{i=1}^{m} y_{i}^{2}=2\left(\begin{array}{c}m+2 \\ 3\end{array}\right)-h^{2}$, and (1) gives

$$
2\left(\begin{array}{c}
3 m+1 \\
3
\end{array}\right)-4\left(\begin{array}{c}
m+2 \\
3
\end{array}\right)=3 \sum_{i=1}^{m} x_{i}^{2}+2(m+1) h^{2} .
$$

The left sides of (3) and (4) reduce to $m\left(25 m^{2}-6 m-7\right) / 3$. Multiplying either of (3) and (4) by 3 and reducing modulo 9 gives the congruence

$$
m\left(25 m^{2}-6 m-7\right) \equiv-3(m-2) h^{2}(\bmod 9) .
$$


For $m \equiv 5,6,7$ or $8(\bmod 9)$, $(5)$ leads to $h^{2} \equiv-1(\bmod 3)$ or another impossibility. For $m \equiv 0$ or $4(\bmod 9)$, $(5)$ implies $h^{2} \equiv 0(\bmod 3)$ and thus $h \equiv 0(\bmod 3)$. For $m \equiv 1$ or $3(\bmod 9),(5)$ gives $h^{2} \equiv 1(\bmod 3)$, so $h \equiv \pm 1(\bmod 3)$. (For $m \equiv 2(\bmod 9)$, (5) is satisfied for any $h$.

Only one example of the second case of Theorem 2 is known: for $m=2$, the set $D=\{(-3,0),(3,0)\}$ covers the $3 \times 6$ board. A computer search shows that there is no other example with $m \leqslant 40$. It seems likely that no other example exists; if for some $m$ there is such a set $D=\left\{\left(x_{i}, y_{i}\right): 1 \leqslant i \leqslant m\right\}$, we have been able to show that $0=\sum x_{i}=\sum x_{i} y_{i}=\sum x_{i}^{2} y_{i}=\sum\left(x_{i}^{3} y_{i}+2 x_{i} y_{i}^{2}\right)$.

By computer search (see the file) we have shown that for all $m$ such that $m \leqslant 40$, $m \neq 3$, and $m \equiv 0,1,2,3$, or $4(\bmod 9)$, there exist sets of $m$ queen squares dominating $Q_{(m+1) \times 3 m}$, with $h$ taking all values not ruled out by Theorem 2 . We believe $g(m)=$ $g^{*}(m)=3 m$ holds for all $m \neq 3$ with $m \equiv 0,1,2,3$, or $4(\bmod 9)$.

The following is immediate from Theorem 2.

Corollary 3. If $m \equiv 5,6,7$, or $8(\bmod 9)$ then $\gamma\left(Q_{(m+1) \times 3 m}\right)=m+1$.

We now examine the cases $m \equiv 5,6,7,8(\bmod 9)$.

Theorem 4. If $m \equiv 5,6$, or $7(\bmod 9)$ then $g^{*}(m) \leqslant 3 m-2$. If $m \equiv 8(\bmod 9)$ then $g^{*}(m) \leqslant 3 m-4$.

Take the board to be a rectangle in the Cartesian plane with sides parallel to the axes, board center at the origin, and squares of edge length two.

For $m \equiv 6$ or $7(\bmod 9)$, assume that $D$ is a set of $m$ squares that dominates the $(m+1) \times(3 m-2)$ board, occupying all but row $h$. If $m \equiv 6(\bmod 9)$ then $h \not \equiv 0(\bmod 3)$. If $m \equiv 7(\bmod 9)$ then $h \equiv 0(\bmod 3)$.

Proof. Let $m$ be a positive integer and $j$ an integer with $0 \leqslant j \leqslant 4$. Let $D$ be a dominating set of $m$ queens for the $(m+1) \times(3 m-j)$-board, with only row $h$ empty.

The $3 m-j$ column indices form the set $S_{\text {col }}=\{j+1-3 m, j+3-3 m, \ldots, 3 m-j-$ $3,3 m-j-1\}$ and the $m+1$ row indices form the set $S_{\text {row }}=\{-m, 2-m, \ldots, m-2, m\}$.

It will be useful to consider row $h$ extended beyond the board, and to look at the congruence classes modulo 3 of the column indices. To this end, for each integer $i$, let $C_{i}^{\prime}=\{x \in \mathbb{Z}: x \equiv i(\bmod 3)\}$. The restriction of $C_{i}^{\prime}$ to column indices of the $(m+1) \times(3 m-j)$-board is $C_{i}=C_{i}^{\prime} \cap S_{\text {col }}$. We write $c_{i}$ for the size of $C_{i}$. By symmetry, $C_{-1}=\left\{-x: x \in C_{1}\right\}=-C_{1}$ so $c_{-1}=c_{1}$.

For each integer $i$ let $R_{i}=\left\{y \in S_{\text {row }}: y \equiv i(\bmod 3)\right\}$. Write $r_{i}$ for the size of $R_{i}$. By symmetry, $R_{-1}=-R_{1}$ so $r_{-1}=r_{1}$.

Lemma 5. Choose $s, b \in\{-1,0,1\}$ such that $s \equiv j$ and $b \equiv k+1$ (mod 3). Then $c_{-1}=c_{1}=c_{0}+s$ and $r_{-1}=r_{1}=r_{0}-b$.

Proof. The facts that $c_{-1}=c_{1}, c_{-1}+c_{0}+c_{1}=3 m-j$ and the $c_{i}$ 's differ by at most one imply the first equation, and the second is similar. 
Let $p$ be the number of squares $(x, y)$ of $D$ with $y \not \equiv h(\bmod 3)$. For each $i \in\{-1,0,1\}$, let $t_{i}$ be the number of squares $(x, y)$ of $D$ such that $x \equiv i(\bmod 3)$ and $y \equiv h(\bmod 3)$. Let $t=t_{-1}+t_{0}+t_{1}$. Thus $|D|=p+t_{-1}+t_{0}+t_{1}=p+t$.

Let $(x, y)$ be a square of $D$. The squares in the extension of row $h$ covered by $(x, y)$ are $(x-(y-h), h),(x, h),(x+(y-h), h)$, some of which may be off the $(m+1) \times(3 m-j)$ board. Their $x$-coordinates $x-(y-h), x, x+(y-h)$ are an arithmetic progression with difference $y-h$. If $y \equiv h(\bmod 3)$ then all of $x-(y-h), x, x+(y-h)$ are in $C_{x}^{\prime}$. If $y \not \equiv h(\bmod 3)$ then the three values $x-(y-h), x, x+(y-h)$ are different modulo 3 , so they contribute one member to each of $C_{-1}^{\prime}, C_{0}^{\prime}, C_{1}^{\prime}$. Thus for each $i \in\{-1,0,1\}, p+3 t_{i}$ is the number of covers (with multiplicity) of squares $(x, h)$ with $x \in C_{i}^{\prime}$.

For each $i \in\{-1,0,1\}$ let $a_{i}$ be the number of "wasted covers" by $D$ of squares $(x, h)$ with $x \in C_{i}^{\prime}$. That is, $a_{i}$ counts every cover of any square $(x, h)$ that is off the board $(|x|>3 m-j-1)$ and all but one cover of each multiply covered square $(x, h)$ on the board. Thus each $a_{i}$ is nonnegative, and since each of the $m$ squares of $D$ covers 3 squares of the extended row $h, a_{-1}+a_{0}+a_{1}=j$.

Since each square in row $h$ of the board is covered by $D$, we get a system of equations:

$$
\begin{aligned}
p+3 t_{-1} & =c_{-1}+a_{-1} \\
p+3 t_{0} & =c_{0}+a_{0} \\
p+3 t_{1} & =c_{1}+a_{1} \\
a_{-1}+a_{0}+a_{1} & =j .
\end{aligned}
$$

As $c_{-1}=c_{1}$, subtracting (6) from (8) shows

$$
a_{-1} \equiv a_{1}(\bmod 3)
$$

For a dominating set $D$ as hypothesized to exist, it is necessary that the total number $t$ of squares $(x, y)$ in $D$ with $y \equiv h(\bmod 3)$ is one less than the number $r_{h}$ of rows in $R_{h}$. When $t=r_{h}-1$, we will say $h$ is eligible for $t$.

Theorem 2 covers the case $j=0$, so we pass to less wide boards, only considering $m \equiv 5,6,7,8(\bmod 9)$.

Let $j=1$. From (10) we have $a_{-1}=a_{1}=0$ and then $a_{0}=1$. Here the $s$ of Lemma 5 is 1 so $c_{-1}=c_{1}=m, c_{0}=m-1$. Then equations (6-8) imply $t_{-1}=t_{0}=t_{1}$, so $t \equiv 0(\bmod 3)$, and the following analysis shows that no $h$ is eligible for any $t$ for $m \equiv 5,6,7,8(\bmod 9)$.

If $m \equiv 5(\bmod 9)$ then $r_{-1}=r_{1}=r_{0}=(m+1) / 3 \equiv-1(\bmod 3)$.

If $m \equiv 6(\bmod 9)$ then $r_{-1}=r_{1}=m / 3 \equiv-1(\bmod 3), r_{0}=(m / 3)+1 \equiv 0(\bmod 3)$.

If $m \equiv 7(\bmod 9)$ then $r_{-1}=r_{1}=(m+2) / 3 \equiv 0(\bmod 3), r_{0}=(m-1) / 3 \equiv$ $-1(\bmod 3)$.

If $m \equiv 8(\bmod 9)$ then $r_{-1}=r_{1}=r_{0}=(m+1) / 3 \equiv 0(\bmod 3)$.

Let $j=2$. Since (9) here implies all $a_{i} \leqslant 2$, (10) gives $a_{-1}=a_{1}$, and then (6-8) imply $t_{-1}=t_{1}$. The $s$ of Lemma 5 is -1 so $c_{-1}=c_{1}=m, c_{0}=m+1$. There are two possibilities:

$\left(a_{-1}, a_{0}, a_{1}\right)=(0,2,0)$, when equations $(6-8)$ imply $t_{0}=t_{1}+1$, so $t \equiv 1(\bmod 3)$, or

$\left(a_{-1}, a_{0}, a_{1}\right)=(1,0,1)$, when equations $(6-8)$ imply $t_{0}=t_{1}$, so $t \equiv 0(\bmod 3)$. 
Then examining the values of the $r_{i}$ 's found above for $m \equiv 5,6,7,8(\bmod 9)$, we see: For $m \equiv 5(\bmod 9)$, all $h$ are eligible for $t \equiv 1(\bmod 3)$ and none for $t \equiv 0(\bmod 3)$; For $m \equiv 6(\bmod 9), h \not \equiv 0(\bmod 3)$ are eligible for $t \equiv 1(\bmod 3)$ and none for $t \equiv$ $0(\bmod 3)$;

For $m \equiv 7(\bmod 9), h \equiv 0(\bmod 3)$ is eligible for $t \equiv 1(\bmod 3)$ and none for $t \equiv$ $0(\bmod 3)$;

For $m \equiv 8(\bmod 9)$, no $h$ is eligible for either $t$.

We continue with $m \equiv 8(\bmod 9)$.

Let $j=3$. Here $c_{-1}=c_{0}=c_{1}=m-1$ so from equations (6-8) we see all $a_{i}$ 's are congruent modulo 3. Either $\left(a_{-1}, a_{0}, a_{1}\right)=(1,1,1)$, and then $t_{-1}=t_{0}=t_{1}$, so $t \equiv 0(\bmod 3)$, or one of the $a_{i}{ }^{\prime}$ s is 3 and the other two are zero, which gives $t \equiv 1(\bmod 3)$. For $m \equiv 8(\bmod 9)$, neither of these gives an eligible $h$, as before.

Let $j=4$. Here there are more possibilities for $\left(a_{-1}, a_{0}, a_{1}\right)$, but the only helpful one for $m \equiv 8(\bmod 9)$ is $(2,0,2)$, which gives $t \equiv-1(\bmod 3)$, with all $h$ eligible.

Computer search reveals (see the file) that for $m \leqslant 40$ and $m \equiv 5,6,7(\bmod 9)$, all minimum dominating sets of $Q_{m+1 \times 3 m-2}$ have just one empty row, and all eligible values of $h$ actually occur. For $m \equiv 8(\bmod 9)$, Theorem 4 does not say any $h$ are ineligible; indeed, our search has found solutions with one empty row for all the $h$ values. The only board size in the $3 \leqslant m \leqslant 40$ range where the minimum dominating sets found have two empty rows is $Q_{9 \times 20}$. Those dominating sets demonstrate numerous patterns of pairs of empty rows, as shown in this file.

We next show that by "pasting together" dominating sets of a certain type, we can extend the range of values for which the bounds of Theorem 4 are known to be exact.

Say that a topless dominating set for the $(m+1) \times n$ board is a dominating set of size $m$ having one square in each row except the top row, which is empty.

\section{Proposition 6.}

(A) Suppose that for $i=1,2$ there is a topless dominating set of $m_{i}$ queens for the $\left(m_{i}+1\right) \times n_{i}$ board. Then there is a dominating set of $m_{1}+m_{2}$ queens for the $\left(m_{1}+m_{2}+1\right) \times\left(n_{1}+n_{2}\right)$ board.

(B) Let $k$ be a positive integer. Suppose that for each $l, 1 \leqslant l \leqslant k$, and for $i=0,1,2$, there exist topless dominating sets of size $9 l+i$ for the $(9 l+i+1) \times(27 l+3 i)$ board. Then for each $m \neq 3,1 \leqslant m \leqslant 9 k+8$, there is a dominating set of size $m$ for the $(m+1) \times(3 m-j)$ board, where $j=0$ if $m \equiv 0,1,2,3$, or $4(\bmod 9), j=2$ if $m \equiv 5,6$, or $7(\bmod 9)$, and $j=4$ if $m \equiv 8(\bmod 9)$.

Proof. We return to our usual scheme of indexing columns and rows from the bottom left board corner. For (A): For $i=1,2$, let $S_{i}$ be a topless dominating set of $m_{i}$ queens for the $\left(m_{i}+1\right) \times n_{i}$ board. On the $\left(m_{1}+m_{2}+1\right) \times\left(n_{1}+n_{2}\right)$ board, the squares in which the columns indexed 1 to $n_{1}$ and the rows indexed 1 to $m_{1}+1$ meet form a copy of the $\left(m_{1}+1\right) \times n_{1}$ board. Place a copy $S_{1}^{\prime}$ of $S_{1}$ on that. The squares in which the columns 
indexed $n_{1}+1$ to $n_{1}+n_{2}$ and the rows indexed $m_{1}+1$ to $m_{1}+m_{2}+1$ meet form a copy of the $\left(m_{2}+1\right) \times n_{2}$ board. Place a copy $S_{2}^{\prime}$ of $S_{2}$, rotated by a half turn, on that. The union of the two copies is a set $S_{1} \oplus S_{2}$ of $m_{1}+m_{2}$ squares that leaves only row $m_{2}+1$ empty on the $\left(m_{1}+m_{2}+1\right) \times\left(n_{1}+n_{2}\right)$ board. As $S_{1}^{\prime}$ covers the left $n_{1}$ squares of that row and $S_{2}^{\prime}$ covers the remainder, $S_{1} \oplus S_{2}$ dominates the $\left(m_{1}+m_{2}+1\right) \times\left(n_{1}+n_{2}\right)$ board.

For (B): We will use topless dominating sets of the $(m+1) \times(3 m-j)$ board for $(m, j)$ $=(1,0),(5,2),(8,4)$, and $(11,0)$. Assume the hypotheses; we then need to prove the existence of (minimum) dominating sets of size $m$ for the $(m+1) \times(3 m-j)$ boards in the ranges claimed. For $m<18$ the database (see the files here and here) contain the claimed dominating sets. For other board sizes of $m=9 l, 9 l+1,9 l+2,2 \leqslant l \leqslant k$, we are assuming there are such sets (in fact, topless). For $m=9 l+3$, we use part (A) to "paste together" topless sets for $m=9 l+2$ and $m=1$. For $m=9 l+4$, we paste together topless sets for $m=9(l-1)+2$ and $m=11$. Pasting together a topless set for each of $m=9 l, 9 l+1,9 l+2$ with a topless set for $m=5$ gives the result for $m=9 l+5,9 l+6,9 l+7$. Finally, pasting together topless sets for $m=9 l$ and $m=8$ gives the result for $m=9 l+8$.

As mentioned after Theorem 2, computer search gave topless dominating sets as required in Proposition 6(B) for $k=4$. It follows that $g^{*}(m)$ equals the bound of Theorem 4 for $m \leqslant 44, m \neq 3$.

We next develop a lower bound for $\gamma\left(Q_{m \times n}\right)$ for more general $m, n$.

Raghavan and Venketesan [18] and Spencer [7, 23] independently proved that

$$
\gamma\left(Q_{n \times n}\right) \geqslant\left\lceil\frac{n-1}{2}\right\rceil .
$$

It has been shown [10] that $\gamma\left(Q_{n \times n}\right)=(n-1) / 2$ only for $n=3,11$. Both of these values are significant for our work here, as we now discuss.

A central queen on $Q_{3 \times 3}$ shows $\gamma\left(Q_{3 \times 3}\right)=1$. This simple fact has a useful generalization: if $C$ is a central sub-board of $Q_{m \times n}$ such that every square of $Q_{m \times n}$ has a line meeting $C$, then a subset of $C$ that occupies all those lines is a dominating set of $Q_{m \times n}$. More than a hundred years ago, Szily [20,21] gave dominating sets of this type for $Q_{13 \times 13}$ and $Q_{17 \times 17}$, which were later shown to be minimum. We found that $Q_{13 \times 16}$ has a minimum dominating set (solution \#23 in the database) of this centrally strong form and have also used this idea to produce good upper bounds of $Q_{m \times n}$ for some $m, n$, as shown below.

It follows from [24] that there are exactly two minimum dominating sets for $Q_{11 \times 11}$. Placing the origin of our coordinate system at board center, these sets are $\mathcal{D}=\{(0,0)$, $\pm(2,4), \pm(4,-2)\}$ (see Figure 3 ) and the reflection of $\mathcal{D}$ across the column $x=0$. So up to equivalence $\mathcal{D}$ is the unique minimum dominating set of $Q_{11 \times 11}$, consisting of a foursome and a queen at its center. This amazing set has an influence on many other values of $\gamma\left(Q_{m \times n}\right)$.

First, since $\mathcal{D}$ fits on $Q_{9 \times 9}$, by omitting edge rows and columns of $Q_{11 \times 11}$ we get dominating sets of $Q_{m \times n}$ for $(m, n)=(10,11),(10,10),(9,11),(9,10),(9,9)$, and these 


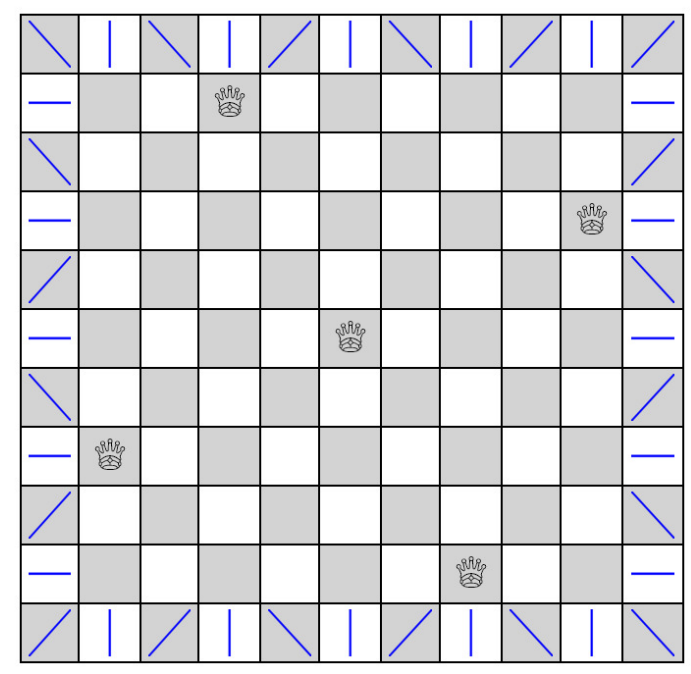

Figure 3: The minimum dominating set $\mathcal{D}$ of $Q_{11 \times 11}$ is shown, with the unique cover of each edge square (see Corollary 8 ) indicated.

turn out to be minimum dominating sets. In a sense, the observed failure of monotonicity, $\gamma\left(Q_{8 \times 11}\right)=6>5=\gamma\left(Q_{9 \times 11}\right)$, occurs simply because $\mathcal{D}$ does not fit on $Q_{8 \times 11}$.

Also, by adding edge rows or columns to $Q_{11 \times 11}$ and adding corner squares to $\mathcal{D}$, we obtain minimum dominating sets for the values $\gamma\left(Q_{11 \times 12}\right)=6, \gamma\left(Q_{11 \times 13}\right)=7, \gamma\left(Q_{12 \times 12}\right)=$ $6, \gamma\left(Q_{12 \times 13}\right)=7, \gamma\left(Q_{12 \times 14}\right)=8, \gamma\left(Q_{13 \times 13}\right)=7, \gamma\left(Q_{13 \times 14}\right)=8, \gamma\left(Q_{14 \times 14}\right)=8$, and $\gamma\left(Q_{15 \times 15}\right)=9$. Finally, it is shown in [25] that $\mathcal{D}$ gives a set implying $\gamma\left(Q_{53 \times 53}\right)=27$.

It was observed by Eisenstein et al. [8] that if a dominating set $D$ of $Q_{n \times n}$ contains no edge squares, the facts that there are $4(n-1)$ edge squares and every queen covers eight edge squares imply $|D| \geqslant\lceil(n-1) / 2\rceil$. This suggests the bound (11).

A similar approach leads one to guess the bound of our next theorem, but some care is needed to handle the general case.

Theorem 7. Let $m, n$ be positive integers with $m \leqslant n$. Then

$$
\gamma\left(Q_{m \times n}\right) \geqslant \min \left\{m,\left\lceil\frac{m+n-2}{4}\right\rceil\right\} .
$$

Proof. It suffices to show that if $\gamma\left(Q_{m \times n}\right) \leqslant m-1$ then $\gamma\left(Q_{m \times n}\right) \geqslant(m+n-2) / 4$. So we assume that $\gamma\left(Q_{m \times n}\right) \leqslant m-1$.

First, suppose $\gamma\left(Q_{m \times n}\right)=m-1$. Then by Proposition 1 we have $n<3 m-2$, which implies $m-1>(m+n-2) / 4$ as needed.

Thus we may take $\gamma\left(Q_{m \times n}\right) \leqslant m-2$ and let $D$ be a minimum dominating set of $Q_{m \times n}$. Since $m \leqslant n$, there are at least two rows and at least two columns that do not contain squares of $D$. Let $a$ be the index of the leftmost empty column, $b$ the index of the rightmost empty column, $c$ the index of the lowest empty row, $d$ the index of 
the highest empty row. The board has a rectangular sub-board $U$ with corner squares $(a, c),(a, d),(b, c)$, and $(b, d)$. Let $E$ be the set of edge squares of this sub-board. We say that $U$ is the box of $D$ and $E$ is the box border of $D$; these sets are defined for any square set $D$ with $|D| \leqslant m-2$. Here $|E|=2(d-c)+2(b-a)$.

Removing columns $a$ and $b$ and rows $c$ and $d$ divides the board into nine regions (some possibly empty). Let $C$ be the set of squares of $D$ inside $U$; that is, $C=\{(x, y) \in$ $D: a<x<b$ and $c<y<d\}$. Let $T_{n w}$ be the set of squares of $D$ in the "northwest" region of the $m \times n$ board; that is, $T_{n w}=\{(x, y) \in D: x<a$ and $y>d\}$. Similarly we label seven more subsets of $D$ by their "geographic direction" from the central region: $T_{n}, T_{n e}, T_{e}, T_{s e}, T_{s}, T_{s w}$, and $T_{w}$. Let $R=T_{n w} \cup T_{n e} \cup T_{s w} \cup T_{s e}$, the set of those squares of $D$ whose orthogonals do not meet $U$. Let $S=T_{n} \cup T_{e} \cup T_{s} \cup T_{w}$, the set of those squares of $D$ having exactly one orthogonal that meets $U$. Then $D$ is the disjoint union of $R, S$, and $C$.

Since each column to the left of column $a$ contains at least one square of $D$,

$$
\left|T_{s w}\right|+\left|T_{w}\right|+\left|T_{n w}\right| \geqslant a-1 .
$$

Similarly,

$$
\begin{aligned}
\left|T_{s e}\right|+\left|T_{e}\right|+\left|T_{n e}\right| & \geqslant n-b \\
\left|T_{s w}\right|+\left|T_{s}\right|+\left|T_{s e}\right| & \geqslant c-1, \\
\left|T_{n w}\right|+\left|T_{n}\right|+\left|T_{n e}\right| & \geqslant m-d .
\end{aligned}
$$

Adding inequalities (13)-(16) and using the definitions of $R$ and $S$ gives

$$
2|R|+|S| \geqslant m+n-2-(d-c)-(b-a) .
$$

Each square in $R$ covers at most two squares of $E$, as the square's orthogonals and one of its diagonals miss $E$. Each square in $S$ covers at most six squares of $E$, as one of the square's orthogonals misses $E$. Each square in $C$ covers eight squares of $E$. Since $D$ is a dominating set, $D$ covers all squares of $E$, so

$$
2|R|+6|S|+8|C| \geqslant 2(d-c)+2(b-a) .
$$

Adding two times (17) to (18) gives

$$
6|R|+8|S|+8|C| \geqslant 2(m+n-2) .
$$

Since $|D|=|R|+|S|+|C|$, adding $2|R|$ to both sides of (19) gives

$$
8|D| \geqslant 2(m+n-2+|R|) \text {. }
$$

Thus

$$
\gamma\left(Q_{m \times n}\right)=|D| \geqslant(m+n-2+|R|) / 4,
$$

which implies the desired conclusion. 


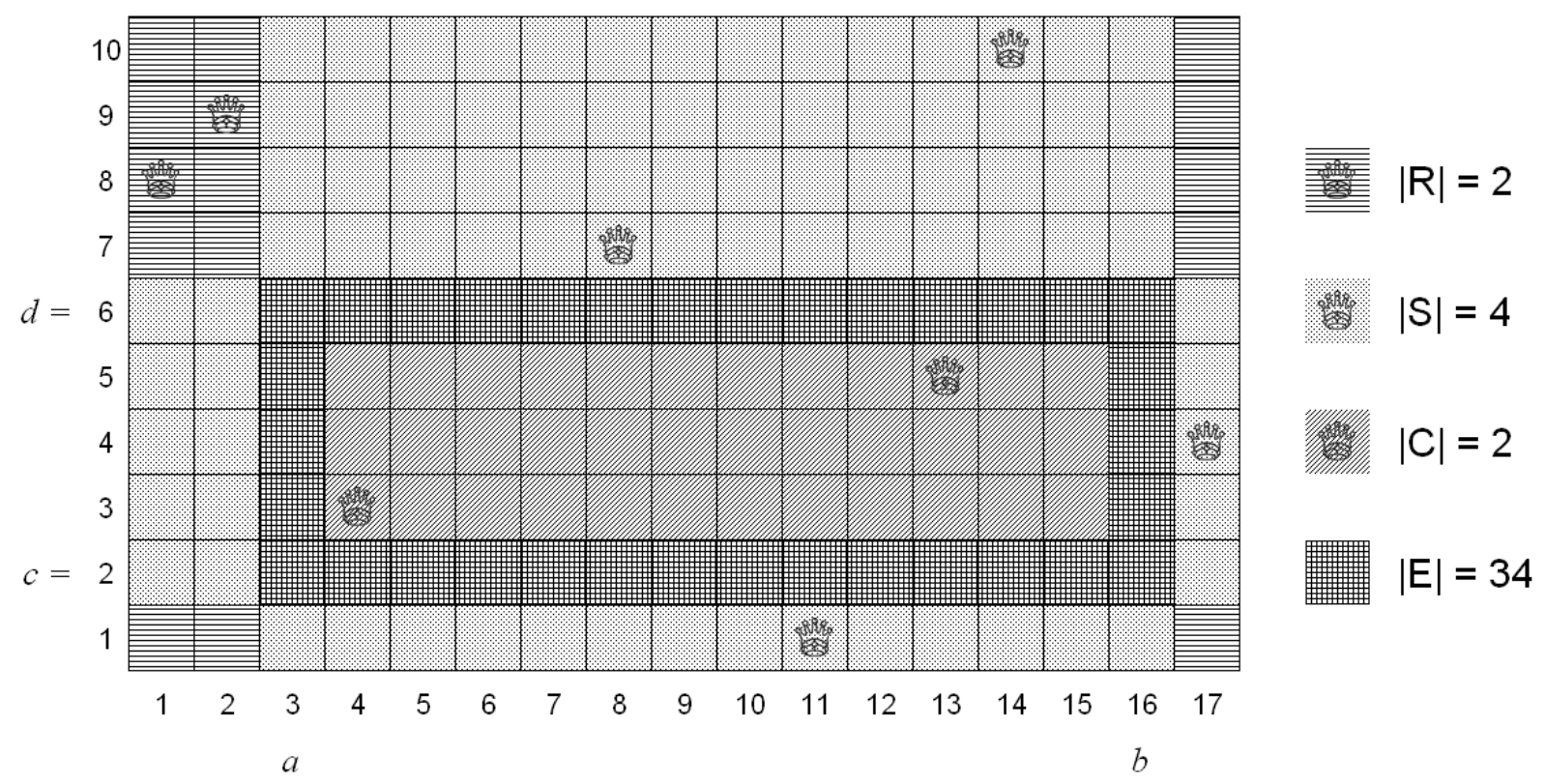

Figure 4: Illustration of the proof of Theorem 7 with $Q_{10 \times 17}: R, S, C$ are the sets of queen squares having respectively 0,1 , or 2 orthogonals meeting the box of $D$, and $E$ is the box border of $D$.

A diagram illustrating the proof for $Q_{10 \times 17}$ is given in Figure 4 .

There are 120 pairs $(m, n)$ satisfying $4 \leqslant m \leqslant n \leqslant 18$. Of these, the bound (12) is achieved for 40 pairs ( 28 with $m \leqslant 6$ ), for 76 pairs the bound is exceeded by one and for the pairs $(12,14),(13,17),(14,16)$, and $(15,15)$ the bound is exceeded by two.

We next explore when $\gamma\left(Q_{m \times n}\right)=(m+n-2) / 4$. From Proposition 1 it follows that for any positive integer $m$, if $n=3 m+2$ then $\gamma\left(Q_{m \times n}\right)=m$, and here $m=(m+n-2) / 4$. So we restrict to $n<3 m+2$.

Corollary 8. Suppose $m \leqslant n<3 m+2$ and $\gamma\left(Q_{m \times n}\right)=(m+n-2) / 4$. Let $D$ be a minimum dominating set of $Q_{m \times n}$. Then $|D| \leqslant m-2$, each box border square is covered exactly once by $D$, and $D$ is independent.

Proof. From $n<3 m+2$ we have $|D|=(m+n-2) / 4 \leqslant m-2$, so the box of $D$ is defined. Since $|D|=(m+n-2) / 4$, in this setting we have equality in inequalities (13)-(20), so each square of the box border $E$ is covered exactly once by $D$. Thus any line meeting $E$ contains at most one square of $D$. Every square of $D$ must be diagonally adjacent to four squares of $E$, so if any line containing a square of $D$ does not meet $E$, it is an orthogonal. From (20) we see that here the set $R$ of "corner squares" in $D$ is empty, so every square of $D$ has at least one orthogonal meeting $E$; then since (13)-(16) are equations here, each orthogonal that misses $E$ contains exactly one square of $D$. Thus $D$ is independent.

Rarely does a minimum dominating set cover each of its box border squares uniquely; see Figure 3 for an example. We also note that the minimum dominating sets \#1-4 
for $Q_{11 \times 12}$ have this property. Each of these sets consists of a foursome centered at $(13 / 2,13 / 2)$ plus the corner squares $(1,1)$ and $(12,1)$, so is not independent.

As mentioned earlier, $\gamma\left(Q_{n \times n}\right)=(n-1) / 2$ is achieved only for $n=3,11$. Considering Corollary 8 , we suspect that the answer to the following question is no.

Question 2. Does $\gamma\left(Q_{m \times n}\right)=(m+n-2) / 4$ with $m \leqslant n<3 m+2$ occur, other than for $(m, n)=(3,3)$ and $(11,11)$ ?

We next extend the method of proof used in $[7,18,23]$ for the lower bound (11) to show that the dimensions of the box of $D$ give a lower bound for $|D|$.

Proposition 9. Let $m \leqslant n$ and let $D$ be a dominating set of $Q_{m \times n}$ of size at most $m-2$. Let $m^{\prime}$ be the number of rows and $n^{\prime}$ the number of columns of the box of $D$. Then:

$$
\begin{aligned}
& \text { If } m^{\prime}>n^{\prime} \text { then }|D| \geqslant\left\lceil\frac{n}{2}\right\rceil ; \\
& \text { If } m^{\prime} \leqslant n^{\prime} \text { then }|D| \geqslant\left\lceil\frac{n-1-\left(n^{\prime}-m^{\prime}\right)}{2}\right\rceil .
\end{aligned}
$$

Proof. Let $a, b, c, d$ be defined as in the proof of Theorem 7. (Then $m^{\prime}=d-c+1$ and $n^{\prime}=b-a+1$.) Since $m^{\prime} \leqslant m \leqslant n$, we may choose an integer $e$ such that $e$ through $e+m^{\prime}-2$ are indices of columns of the board. Set $S=\left\{(x, c),(x, d): e \leqslant x \leqslant e+m^{\prime}-2\right\}$ and $P=\left\{(x, y) \in D: x<e\right.$ or $\left.x>e+m^{\prime}-2\right\}$. Then no square is diagonally adjacent to more than two squares of $S$ and no square of $P$ is orthogonally adjacent to any square of $S$. As the $2\left(m^{\prime}-1\right)$ squares of $S$ are covered by $D, 2\left(m^{\prime}-1\right) \leqslant 2|P|+4(|D|-|P|)$, which implies

$$
|D| \geqslant\left\lceil\frac{m^{\prime}-1+|P|}{2}\right\rceil .
$$

If $m^{\prime}>n^{\prime}$ then we can choose $e$ so that all columns that do not meet $S$ are occupied, so $|P| \geqslant n-\left(m^{\prime}-1\right)$. If $m^{\prime} \leqslant n^{\prime}$ we can choose $e$ so that $S$ is contained in the top and bottom edges of $U$, and then $|P| \geqslant n-n^{\prime}$. In both cases, (21) implies the conclusion.

As $\gamma\left(Q_{n \times n}\right)=(n-1) / 2$ only for $n=3,11$, we have $\gamma\left(Q_{n \times n}\right) \geqslant\lceil n / 2\rceil$ for all other positive integers $n$. There is much evidence that this lower bound is quite good. Work from $[4,5,11,14,17,23,24]$ reported in [17] shows that for $n$ from 1 to 120, excluding 3 and 11 , we have $\lceil n / 2\rceil \leqslant \gamma\left(Q_{n \times n}\right) \leqslant\lceil n / 2\rceil+1$. In this range, $\gamma\left(Q_{n \times n}\right)=\lceil n / 2\rceil$ is known for 46 values of $n$ and $\gamma\left(Q_{n \times n}\right)=\lceil n / 2\rceil+1$ is known for $n=8,14,15,16$. Also, $\gamma\left(Q_{(4 k+1) \times(4 k+1)}\right)=2 k+1$ is known for $1 \leqslant k \leqslant 32$.

For $m<n$, we have little evidence that the bound (12) is good. We were not able to use the methods of the proofs of Theorem 7 and Proposition 9 to improve on this bound. Also, a computer search using a greedy algorithm for some larger $m, n$ did not supply evidence about lower bounds for $\gamma\left(Q_{m \times n}\right)$.

The statement of Proposition 9 leads one to consider the quantity $n / 2$. We have checked that when $4 \leqslant m \leqslant n \leqslant 18, \gamma\left(Q_{m \times n}\right) \geqslant \min \{m-1,\lfloor n / 2\rfloor-1\}$. This bound and the bound (12) are close only when $m$ and $n$ are close. So we ask the following.

Question 3. For $m, n$ with $m \leqslant n$, what is a good general lower bound for $\gamma\left(Q_{m \times n}\right)$ ? In particular, is it true that $\gamma\left(Q_{m \times n}\right) \geqslant \min \{m-1,\lfloor n / 2\rfloor-1\}$ ? 


\section{Construction of dominating sets}

Given dimensions $m$ and $n$, we would like a general approach that would allow us to construct minimum dominating sets of $Q_{m \times n}$, or at least reasonably small dominating sets. We have two difficulties to consider.

The first difficulty was just discussed: in general we know the value of $\gamma\left(Q_{m \times n}\right)$ only approximately.

The second difficulty is that construction of a dominating set of $Q_{m \times n}$ generally means specifying most or all of the lines that the set is to occupy. There are some restrictions that the indices of the lines must satisfy, as we now describe.

Let $D=\left\{\left(x_{i}, y_{i}\right): 1 \leqslant i \leqslant l\right\}$ be a set of $l$ squares of $Q_{m \times n}$ that occupies difference diagonals $\left(d_{i}\right)_{i=1}^{l}$ and sum diagonals $\left(s_{i}\right)_{i=1}^{l}$. Since the square $(x, y)$ is on the difference diagonal with index $y-x$ and the sum diagonal with index $y+x$, summing over $D$ gives

$$
\sum_{i=1}^{l} d_{i}=\sum_{i=1}^{l} y_{i}-\sum_{i=1}^{l} x_{i} \text { and } \sum_{i=1}^{l} s_{i}=\sum_{i=1}^{l} y_{i}+\sum_{i=1}^{l} x_{i} .
$$

The Parallelogram Law $2 x^{2}+2 y^{2}=(y-x)^{2}+(y+x)^{2}$ gives a quadratic constraint

$$
2 \sum_{i=1}^{l} x_{i}^{2}+2 \sum_{i=1}^{l} y_{i}^{2}=\sum_{i=1}^{l} d_{i}^{2}+\sum_{i=1}^{l} s_{i}^{2}
$$

on the line indices.

In each of the two constructions given below, we will refer to lines that must be occupied for domination as required lines and other lines as auxiliary lines.

Both constructions produce a number of minimum dominating sets, but neither can produce a dominating set of $Q_{m \times n}$ of size less than $\lfloor n / 2\rfloor$. This is a little evidence for the possible bound mentioned in Question 3.

\subsection{Domination by orthodox covers}

This idea generalizes [25, Section 2]. Let $D$ be a set of squares of $Q_{m \times n}$. If it is possible to place the origin of the coordinate system so that every even column and every even row contains a square of $D$, we will say $D$ is an orthodox set. That is, an orthodox set is one that occupies at least every other column and every other row of $Q_{m \times n}$.

Say that square $(x, y)$ of $Q_{m \times n}$ is even if $x+y$ is even, odd if $x+y$ is odd. We divide the even squares of $Q_{m \times n}$ into two classes: $(x, y)$ is even-even if both $x$ and $y$ are even, odd-odd if both are odd. If $D$ is an orthodox set and each odd-odd square of $Q_{m \times n}$ is covered by some square of $D$, we say $D$ is an orthodox cover. For example, solution \#10 for $Q_{7 \times 11}$ given in Table 1 is an orthodox cover; take the origin at $(6,3)$ to see this.

It is clear from the definition that an orthodox cover dominates every even square of $Q_{m \times n}$, and since every odd square of $Q_{m \times n}$ is on one even-indexed orthogonal, all odd squares are also dominated: an orthodox cover is a dominating set of $Q_{m \times n}$. Many orthodox covers appear in the appendix, and are labeled there as such. 
Since $Q_{m \times n}$ has at least $\lfloor n / 2\rfloor$ even-indexed columns, an orthodox set on $Q_{m \times n}$ has at least $\lfloor n / 2\rfloor$ members. Generally, we expect that most of the squares of $D$ will be eveneven, to help dominate the odd-odd squares diagonally. When $n$ is considerably larger than $m$, there are more possibilities of placing queens on odd squares that occupy even columns. Also, it is possible sometimes to achieve a dominating set of size less than $\lfloor n / 2\rfloor$ by a minor modification, as is shown in solution $\# 1$ for $Q_{7 \times 12}$ in Table 1 . If the center of the square there labeled $(6,3)$ is taken to be the origin of the coordinate system, the dominating set shown misses being an orthodox set only by not occupying the rightmost column. Thus the three odd-odd squares in that column are not covered along their column, as they would be by an orthodox set. But the queen on a dark square covers those three squares, and the odd squares of its column, and thus completes a dominating set of size 5 .

Minimum dominating set \#147 for $Q_{12 \times 16}$ is an orthodox cover with a single queen on a dark square, at $(6,10)$. The squares covered only by this queen are the dark squares in its column and $(1,10)$. Replacing $(6,10)$ with the white square $(6,5)$ covers those squares and, adding a row 0 to the board, also the square $(1,0)$. In fact, the full set now covers all of row 0 and is thus a minimum dominating set of $Q_{13 \times 16}$; it is solution \#15 for $Q_{13 \times 16}$, rotated by a half-turn.

There are many ways to create orthodox covers, and we will only give one example. An approach is to regard $Q_{m \times n}$ as the union of overlapping copies of $Q_{m \times m}$; for odd $m$, this allows us to use [25, Theorem 1], which gives sufficient conditions for an orthodox set on $Q_{m \times m}$ to be an orthodox cover.

Example 1. An orthodox cover implying $\gamma\left(Q_{13 \times 19}\right) \leqslant 10$.

We take the origin of the coordinate system to be the center of $Q_{13 \times 19}$, and regard $Q_{13 \times 19}$ as the union of two copies of $Q_{13 \times 13}$, centered at $( \pm 3,0)$. From [25, Theorem 1], if we regard the center of $Q_{13 \times 13}$ as the origin, an orthodox set on $Q_{13 \times 13}$ dominates if the set occupies the sum and difference diagonals with indices in $\{-6,-2,0,2,6\}$. Asking this on both copies of $Q_{13 \times 13}$, we wish to have our orthodox set occupy the sum and difference diagonals which (on $Q_{13 \times 19}$ ) have indices in $\{-6,-2,0,2,6\} \pm 3$, which is $\{ \pm 1, \pm 3, \pm 5, \pm 9\}$, so there will be two auxiliary difference diagonal indices $d_{1}, d_{2}$ and two auxiliary sum diagonal indices $s_{1}, s_{2}$. The required column indices are $\pm 1, \pm 3, \pm 5, \pm 7, \pm 9$, so there will be no auxiliary column indices. The required row indices are $0, \pm 2, \pm 4, \pm 6$, so there will be three auxiliary row indices $r_{1}, r_{2}, r_{3}$.

From (22) we have $d_{1}+d_{2}=r_{1}+r_{2}+r_{3}=s_{1}+s_{2}$ and (23) gives $d_{1}^{2}+d_{2}^{2}+s_{1}^{2}+$ $s_{2}^{2}=420+2\left(r_{1}^{2}+r_{2}^{2}+r_{3}^{2}\right)$. We attempt to find a solution with symmetry by a halfturn about the board center: this means $r_{1}=0, r_{2}=-r_{3}, d_{1}=-d_{2}$, and $s_{1}=-s_{2}$. Then the quadratic constraint simplifies to $d_{1}^{2}+s_{1}^{2}=210+2 r_{2}^{2}$, of which one solution is $d_{1}=13, s_{1}=7, r_{2}=2$. Now all lines are specified, and it is not difficult to find the solution $D=\{ \pm(9,0), \pm(7,-6), \pm(5,2), \pm(3,2), \pm(1,-4)\}$. 


\subsection{Domination by centrally strong sets}

We begin by considering a board $C$ which is to be a central sub-board of a larger board $B$. Say that $C$ has $m_{1}$ rows and $n_{1}$ columns, with $m_{1} \geqslant n_{1}$ and $m_{1}, n_{1}$ not both even. It is convenient here to have the board squares of side length two, and place $C$ with its center at the origin. Thus if, for example, $m_{1}$ is odd and $n_{1}$ is even, then each square has center $(x, y)$ with $x$ an odd integer and $y$ an even one.

We then wish to choose a nonnegative integer $k$ and a set $D$ of squares of $B$ (actually, all or almost all in $C$ ) such that $D$ contains at least one square from the extension of each orthogonal of $C$ to $B$, and $D$ contains exactly one square from the extension of each difference diagonal of $C$, except none from the highest $k$ and the lowest $k$ extended difference diagonals; similarly for sum diagonals. Let

$$
m=m_{1}+2 n_{1}-2 k, \quad n=2 m_{1}+n_{1}-2 k, \quad g=m_{1}+n_{1}-2 k-1 .
$$

Then $m \leqslant n$, and it is straightforward to verify that if $C$ is taken to be the central $m_{1} \times n_{1}$ sub-board of the $m \times n$ board $B$, then $D$ is a dominating set of $Q_{m \times n}$ and $|D|=g$. Such a $D$ will be called a centrally strong set, as it generalizes the idea discussed for square boards in [25, page 234]. We note that our definition requires each square of $D$ to have both diagonals among the required ones, and thus both have indices of absolute value at most $m_{1}+n_{1}-2 k-2$, but this does not imply $D \subseteq C$. If in fact $D \subseteq C$, we say that $D$ is a strict centrally strong set.

A number of strict centrally strong sets occur in the appendix, and are labeled there as such. We note that these sets can only occur when $m \leqslant n<2 m$; this follows from (24) and the fact that since there will be $n_{1}-2 k-1$ auxiliary row indices, this quantity is nonnegative.

One merit of this construction is that a single centrally strong $D$ gives an upper bound for $\gamma\left(Q_{m \times n}\right)$ for several pairs $(m, n)$ since $D$ is confined to a small central region of the $m \times n$ board, especially if $D$ is strict. For example, there is a strict centrally strong set $D=\{ \pm(-5,0), \pm(-3,4), \pm(-1,6), \pm(1,2), \pm(3,6)\}$ with $m_{1}=9, n_{1}=6$, and $k=2$, and $|D|=10$, which shows that $\gamma\left(Q_{m \times n}\right) \leqslant 10$ when $9 \leqslant m \leqslant 17$ and $6 \leqslant n \leqslant 20$. For some of these pairs $(m, n)$, this bound is poor, but for the six pairs with $m+n \geqslant 35$, combining with the bound (12) gives $9 \leqslant \gamma\left(Q_{m \times n}\right) \leqslant 10$, and 10 is a useful upper bound for some of the smaller boards also.

The simplest centrally strong sets occur with $m_{1} \geqslant 1, n_{1}=1$ and $k=0$, where we get $m_{1}$ queens occupying all squares of the $m_{1} \times 1$ board $C$, and the following bound (which we have stated in terms of $\left.m=m_{1}+2\right)$. For $3 \leqslant m \leqslant 10$ at least, this bound gives the exact value of $\gamma\left(Q_{m \times(2 m-3)}\right)$.

Proposition 10. For $m \geqslant 3, \gamma\left(Q_{m \times(2 m-3)}\right) \leqslant m-2$.

We next consider the effect of (22) and (23) on the search for centrally strong sets. Symmetry and the requirement that each difference diagonal contains exactly one square of $D$ imply that the sum of the difference diagonal indices of $D$ is zero. Similarly the sum of the sum diagonal indices of $D$ is zero, and then (22) implies that $\sum_{(x, y) \in D} x=0$ and 
$\sum_{(x, y) \in D} y=0$. As we require a centrally strong set to occupy all (extended) columns of the sub-board, we regard the $n_{1}$ indices of these columns as required column indices; by symmetry their sum is zero. As $C$ has $n_{1}$ columns and $g$ occupied squares, there will be $g-n_{1}=m_{1}-2 k-1$ auxiliary column indices, each having parity opposite to that of $n_{1}$. Since $\sum_{(x, y) \in D} x=0$ and all required column indices sum to zero, so do the auxiliary column indices. Similarly there will be $m_{1}$ required row indices and $g-m_{1}=n_{1}-2 k-1$ auxiliary row indices, with sum zero, each having parity opposite to that of $m_{1}$. If $D$ is strict, then all indices of occupied columns have absolute value at most $n_{1}-1$ and all indices of occupied rows have absolute value at most $m_{1}-1$. (We have required that $m_{1}, n_{1}$ not both be even because if they were, there would be an odd number of auxiliary row indices, each odd, so their sum could not be even, thus not zero.)

Using the identities $\sum_{i=1}^{j}(2 i-1)^{2}=\left(\begin{array}{c}2 j+1 \\ 3\end{array}\right)$ and $\sum_{i=1}^{j}(2 i)^{2}=\left(\begin{array}{c}2 j+2 \\ 3\end{array}\right)$, we see that the sum of the squares of the indices of all occupied diagonals of $C$ is $4\left(\begin{array}{c}g+1 \\ 3\end{array}\right)$, the sum of the squares of the required column indices is $2\left(\begin{array}{c}n_{1}+1 \\ 3\end{array}\right)$ and the sum of the squares of the required row indices is $2\left(\begin{array}{c}m_{1}+1 \\ 3\end{array}\right)$. Letting $\sum_{\text {orth }}$ denote the sum of the squares of the auxiliary column indices and auxiliary row indices, the quadratic constraint (23) gives

$$
\sum_{\text {orth }}=2\left[\left(\begin{array}{c}
g+1 \\
3
\end{array}\right)-\left(\begin{array}{c}
m_{1}+1 \\
3
\end{array}\right)-\left(\begin{array}{c}
n_{1}+1 \\
3
\end{array}\right)\right]
$$

Combined with Proposition 10, part (a) of the following proposition shows how small a centrally strong set can be. In parts (b) and (c), we limit the values of $m_{1}, n_{1}, k$ that need be considered when constructing centrally strong sets.

We say that a value of $k$ for which there exists a centrally strong set on $Q_{m_{1} \times n_{1}}$ is feasible for $\left(m_{1}, n_{1}\right)$.

\section{Proposition 11.}

(a) For any centrally strong set $D$ with $n_{1}>1,|D| \geqslant n / 2$.

(b) For any $\left(m_{1}, n_{1}\right)$, it is only necessary to use the largest feasible $k$ to determine all upper bounds for $\gamma\left(Q_{m \times n}\right)$ implied by centrally strong sets from $\left(m_{1}, n_{1}\right)$.

(c) If $k$ is feasible for $\left(m_{1}, n_{1}\right)$ and $k+1$ is feasible for $\left(m_{1}, n_{1}+2\right)$, the latter gives the more useful result.

Proof. (a): As the number $n_{1}-2 k-1$ of auxiliary row indices is nonnegative, $n_{1} \geqslant 2 k+1$. If $n_{1}=2 k+1$ then $g=m_{1}$ by (24), and then the fact that the right side of (25) is nonnegative implies $n_{1}=1$ and $k=0$, the situation of Proposition 10. Thus for $k \geqslant 1$ we have $n_{1} \geqslant 2 k+2$, which by (24) is equivalent to $|D| \geqslant n / 2$.

(b): Suppose for some integer $h>0$ that both $k$ and $k-h$ are feasible for $\left(m_{1}, n_{1}\right)$. Then the triple $m_{1}, n_{1}, k$ gives a dominating set $D$ of size $g$ on $Q_{m \times n}$, where $m, n, g$ are determined by (24), and similarly the triple $m_{1}, n_{1}, k-h$ gives a dominating set $D^{\prime}$ of size $g+2 h$ on $Q_{(m+2 h) \times(n+2 h)}$. However, by repeating $2 h$ times the process of adding an edge 
row and edge column to the board and the new corner square to the dominating set, we can construct from $D$ a dominating set of $Q_{(m+2 h) \times(n+2 h)}$ of the same size as $D^{\prime}$.

(c): Using (24), if $m_{1}, n_{1}, k$ gives a dominating set of size $g$ for $Q_{m \times n}$, then $m_{1}, n_{1}+$ $2, k+1$ gives a dominating set of size $g$ for $Q_{(m+2) \times n}$.

Example 2. A centrally strong set implying $\gamma\left(Q_{13 \times 16}\right) \leqslant 8$.

Let $m_{1}=7$ and $n_{1}=4$, and $k=1$. Then a strict centrally strong set $D$ is to have one auxiliary row index, which from $\sum_{(x, y) \in D} y=0$ must be zero, and two auxiliary column indices, say $c_{1}, c_{2}$, each in $\{-3,-1,1,3\}$. From $\sum_{(x, y) \in D} x=0$ we see $c_{2}=-c_{1}$ and from (25) we have $c_{1}^{2}+c_{2}^{2}=18$, so we can take $c_{1}=3$ and $c_{2}=-3$. We then easily obtain $D=\{ \pm(1,-6), \pm(3,4), \pm(3,0), \pm(3,-2)\}$; see solution \#23 for $Q_{13 \times 16}$. (Recall that board squares have edge length two, column indices are even integers, and row indices are odd integers here.) Using (24) this gives $\gamma\left(Q_{13 \times 16}\right) \leqslant 8$ (and equality holds by our computer search).

We give two infinite families of strict centrally strong sets, each including a minimum dominating set found by von Szily [20, 21].

Example 3. Strict centrally strong sets for $n_{1}=5, k=1$ and odd $m_{1} \geqslant 5$, and for $n_{1}=7, k=2$ and odd $m_{1} \geqslant 7$.

In our approach described above, all orthogonal indices would be even here; we have divided by two, thus returning to a board with squares of edge length one.

For $n_{1}=5, k=1$, and $m_{1} \equiv 1(\bmod 4), D$ consists of $\pm\left(-1, \frac{m_{1}-1}{2}\right),(0,0)$, and $\pm(0,2 i)$ and $\pm\left(2, \frac{m_{1}+5}{2}-4 i\right)$ for $1 \leqslant i \leqslant \frac{m_{1}-1}{4}$. With $m_{1}=5$, this gives a minimum dominating set of $Q_{13 \times 13}$ found by von Szily [20]; see also solution \#41.

For $n_{1}=5, k=1$, and $m_{1} \equiv-1(\bmod 4), D$ consists of $\pm\left( \pm 1, \frac{m_{1}-1}{2}\right), \pm\left(-1, \frac{m_{1}-3}{2}\right)$, $(0,0), \pm(0,2 i)$ for $1 \leqslant i \leqslant \frac{m_{1}-7}{4}$, and $\pm\left(2, \frac{m_{1}+3}{2}-4 i\right)$ for $1 \leqslant i \leqslant \frac{m_{1}-3}{4}$.

These sets show that for $i \geqslant 3$, if $2 i-1 \leqslant m \leqslant 2 i+7$ and $5 \leqslant n \leqslant 4 i+1$, then $\gamma\left(Q_{m \times n}\right) \leqslant 2 i+1$.

Now let $n_{1}=7$.

For $m_{1}=7$, let $D=\{i(1,2)+j(2,-1):-1 \leqslant i, j \leqslant 1\}$. This gives a minimum dominating set of $Q_{17 \times 17}$ found by von Szily [21]; see also solution \#21.

For $m_{1}=9$, let $D=\{(0,0), \pm(1,4), \pm(2,-3), \pm(1,2)+j(2,-1):-1 \leqslant j \leqslant 1\}$. This gives $\gamma\left(Q_{19 \times 21}\right) \leqslant 11$, which is the best we know.

The following complicated description of a placement is the result of unifying four cases depending on the residue of $m_{1}$ modulo 8. Any odd $m_{1} \geqslant 11$ has a unique expression $m_{1}=$ $11+2\left(l_{1}+l_{2}\right)$ with $l_{1}$ an integer and either $l_{2}=l_{1}$ or $l_{2}=l_{1}+1$. (Here $l_{1}=\left\lfloor\left(m_{1}-11\right) / 4\right\rfloor$ and $l_{2}=\left\lceil\left(m_{1}-11\right) / 4\right\rceil$.)

Start with $(0,0), \pm(1,2), \pm(2,-3), \pm(3,-1), \pm\left((-1)^{l_{1}},-2 l_{1}-5\right), \pm\left((-1)^{l_{2}+1},-2 l_{2}-4\right)$. $A d d \pm(2,4 j)$ and $\pm(2,4 j+1)$ for $1 \leqslant j \leqslant\left\lceil l_{2} / 2\right\rceil$, and add $\pm(2,-4 j-2)$ and $\pm(2,-4 j-3)$ for $1 \leqslant j \leqslant\left\lfloor l_{2} / 2\right\rfloor$. If $l_{2}=l_{1}$ then add $\pm\left(2,(-1)^{l_{1}}\left(2 l_{1}+4\right)\right)$.

These sets show that for $i \geqslant 4$, if $2 i-1 \leqslant m \leqslant 2 i+9$ and $7 \leqslant n \leqslant 4 i+1$, then $\gamma\left(Q_{m \times n}\right) \leqslant 2 i+1$. 
Above we have described two approaches to the construction of dominating sets. In both, once a set of lines to be occupied by the dominating set is specified, it is necessary to see whether one can find such a dominating set. A fast backtrack search idea of Hitotumatu and Noshita [12], explained and amplified by Knuth [15], was used by Östergård and Weakley [17] to find values and bounds of $\gamma\left(Q_{n \times n}\right)$ up to $n=120$. This approach and also the algorithm of Neuhaus [16] can be applied to rectangular boards as well. But as mentioned, neither of our constructions can produce a dominating set of size less than $\lfloor n / 2\rfloor$ for $Q_{m \times n}$ (with $m \leqslant n$ ). Thus a resolution of Question 3 would be needed to determine whether extensive search based on these constructions is useful.

The complexity of computing minimum dominating set of queens is another open question [9, Section 5]. Backtracking algorithms, dynamic programming, and treewidth technique are analyzed extensively by Fernau [9, Sections 2-4].

Applications of backtracking algorithms to a variety of domination problems are studied in the doctoral dissertation of Bird [3]; in particular, he examines how recursive backtracking search can be split among multiple processes by partitioning the search tree. We give some of his results on queens at the end of the next section.

\section{Independent domination}

We have calculated the independent domination number $i\left(Q_{m \times n}\right), 4 \leqslant m \leqslant n \leqslant 18$, as shown in the table. Each table entry is linked to some minimum independent dominating sets. In these ranges for $m$ and $n$, monotonicity fails twice: $i\left(Q_{8 \times 11}\right)=6>5=i\left(Q_{9 \times 11}\right)=$ $i\left(Q_{10 \times 11}\right)=i\left(Q_{11 \times 11}\right)$, and $i\left(Q_{11 \times 18}\right)=9>8=i\left(Q_{12 \times 18}\right)$. The first instance is essentially the same failure as for $\gamma\left(Q_{m \times n}\right)$. The second is similar in that the only (up to symmetry) independent dominating set of size 8 for $Q_{12 \times 18}$ does not fit on $Q_{11 \times 18}$.

From the definitions it is clear that $\gamma\left(Q_{m \times n}\right) \leqslant i\left(Q_{m \times n}\right)$, and this appears to be an excellent lower bound for $i\left(Q_{m \times n}\right)$. In the table, we have highlighted the entries where these two numbers are unequal. We know of no case where $\gamma\left(Q_{m \times n}\right)+1<i\left(Q_{m \times n}\right)$.

Bird [3, Chapter 5] reports the new values $i\left(Q_{n \times n}\right)=\gamma\left(Q_{n \times n}\right)=(n / 2)+1$ for $n=$ $20,22,24, i\left(Q_{19 \times 19}\right)=i\left(Q_{21 \times 21}\right)=11$, and $i\left(Q_{23 \times 23}\right)=13$. He also gives the number of minimum dominating sets and the number of minimum independent dominating sets, up to equivalence, for $Q_{n \times n}$ up to $n=18$.

\section{Acknowledgements}

The authors thank the anonymous reviewers for their substantial comments. Research of the first author was supported in part by the Hungarian Scientific Research Fund (OTKA), grant no. K111797, and the János Bolyai Research Fellowship of the Hungarian Academy of Sciences, grant no. BO/00154/16, and the ÚNKP-18-4-BCE-90 Bolyai+ New National Excellence Program of the Ministry of Human Capacities, Hungary.

Parts of the computations were carried out on NIIF Supercomputer based in Hungary at NIIF National Information Infrastructure Development Institute. 


\begin{tabular}{|c||c|c|c|c|c|c|c|c|c|c|c|c|c|c|c|}
\hline$n \backslash m$ & 4 & 5 & 6 & 7 & 8 & 9 & 10 & 11 & 12 & 13 & 14 & 15 & 16 & 17 & 18 \\
\hline \hline 4 & 3 & & & & & & & & & & & & & & \\
\hline 5 & 3 & 3 & & & & & & & & & & & & & \\
\hline 6 & 3 & 3 & 4 & & & & & & & & & & & & \\
\hline 7 & 3 & 4 & 4 & 4 & & & & & & & & & & & \\
\hline 8 & 4 & 4 & 4 & 5 & 5 & & & & & & & & & & \\
\hline 9 & 4 & 4 & 4 & 5 & 5 & 5 & & & & & & & & & \\
\hline 10 & 4 & 4 & 4 & 5 & 5 & 5 & 5 & & & & & & & & \\
\hline 11 & 4 & 4 & 5 & 5 & 6 & 5 & 5 & 5 & & & & & & & \\
\hline 12 & 4 & 4 & 5 & 5 & 6 & 6 & 6 & 6 & 7 & & & & & & \\
\hline 13 & 4 & 5 & 5 & 6 & 6 & 6 & 7 & 7 & 7 & 7 & & & & & \\
\hline 14 & 4 & 5 & 6 & 6 & 6 & 6 & 7 & 7 & 8 & 8 & 8 & & & & \\
\hline 15 & 4 & 5 & 6 & 6 & 7 & 7 & 7 & 7 & 8 & 8 & 9 & 9 & & & \\
\hline 16 & 4 & 5 & 6 & 6 & 7 & 7 & 7 & 8 & 8 & 8 & 9 & 9 & 9 & & \\
\hline 17 & 4 & 5 & 6 & 7 & 7 & 7 & 8 & 8 & 8 & 9 & 9 & 9 & 9 & 9 & \\
\hline 18 & 4 & 5 & 6 & 7 & 7 & 8 & 8 & $\mathbf{9}$ & 8 & 9 & 9 & 9 & 10 & 10 & 10 \\
\hline
\end{tabular}

Table 2: Values of independent domination number $i\left(Q_{m \times n}\right), 4 \leqslant m \leqslant n \leqslant 18$ (OEIS A299029). Highlighted cells indicate where $\gamma \neq i$.

\section{References}

[1] W. Ahrens. Mathematische Unterhaltungen und Spiele. B.G. Teubner, Leipzig-Berlin, 1901.

[2] W. Ahrens. Mathematische Unterhaltungen und Spiele. B.G. Teubner, Leipzig-Berlin, 1910.

[3] W. Bird. Computational Methods for Domination Problems. Doctoral dissertation, University of Victoria, 2017.

[4] A.P. Burger and C.M. Mynhardt. Symmetry and domination in queens graphs. Bull. Inst. Combin. Appl., 29:11-24, 2000.

[5] A.P. Burger, C.M. Mynhardt, and E.J. Cockayne. Domination numbers for the queen's graph. Bull. Inst. Combin. Appl., 10:73-82, 1994.

[6] G. Chartrand, T.W. Haynes, M.A. Henning, and P. Zhang. From Domination to Coloring. In: Pioneer of Domination in Graphs, SpringerBriefs in Mathematics. Springer, Cham, Chapter 1, pp. 1-13, 2019.

[7] E.J. Cockayne. Chessboard domination problems. Discrete Math. 86:13-20, 1990.

[8] M. Eisenstein, C. Grinstead, B. Hahne, and D. van Stone. The queen domination problem. In Proceedings of the Twenty-third Southeastern International Conference on Combinatorics, Graph Theory, and Computing, Congr. Numer. 91:189-193, 1992.

[9] H. Fernau. Minimum dominating set of queens: A trivial programming exercise?, Discrete Appl. Math., 158:308-318, 2010. 
[10] D. Finozhenok and W.D. Weakley. An improved lower bound for domination numbers of the queen's graph. Australas. J. Combin., 37:295-300, 2007.

[11] P.B. Gibbons and J. Webb. Some new results for the queens domination problem. Australas. J. Combin. 15:145-160, 1997.

[12] H. Hitotumatu and K. Noshita. A technique for implementing backtrack algorithms and its application. Inform. Process. Lett., 8:174-175, 1979.

[13] C.F. de Jaenisch. Traité des Applications de l'Analyse Mathématique au Jeu des Échecs, Appendix, pages $244 \mathrm{ff}$ (Imperial St. Petersburg Academy of Sciences, St. Petersburg, 1862).

[14] M.D. Kearse and P.B. Gibbons. Computational methods and new results for chessboard problems. Australas. J. Combin. 23:253-284, 2001.

[15] D.E. Knuth. Dancing links. In Millennial Perspectives in Computer Science, J. Davies, B. Roscoe, and J. Woodcock (Ed.), 187-214 (Palgrave, Houndmills, 2000).

[16] S. Neuhaus. Eigenschaften kleinster dominierender Mengen und Dominanzzahlen von Damengraphen. Faculty of Mathematics $\&$ Natural Sciences, Univ. Cologne (2009).

[17] P.R.J. Östergård and W.D. Weakley. Values of domination numbers of the queen's graph. Electron. J. Combin., 8(1): Research Paper 29, 2001.

[18] V. Raghavan and S.M. Venketesan. On bounds for a covering problem. Inform. Process. Lett., 25:281-284, 1987.

[19] W.W. Rouse Ball. Mathematical Recreations 85 Essays, 3rd Edition, Chapter 6, Minimum pieces problem (Macmillan, London, 1939).

[20] K. von Szily, Das Minimalproblem der Damen, Deutsche Schachzeitung, 57(1902), 326-328.

[21] K. von Szily, Das Minimalproblem der Damen, Deutsche Schachzeitung, 58(1903), 65-68.

[22] J.J. Watkins. Across the Board: The Mathematics of Chessboard Problems, Chapter 8 (Princeton University Press, Princeton and Oxford, 2004).

[23] W.D. Weakley. Domination in the queen's graph. In Graph Theory, Combinatorics, and Algorithms, Vol. 2 (Kalamazoo, MI 1992), Y. Alavi and A. Schwenk (Editors), 1223-1232 (Wiley, New York, 1995).

[24] W.D. Weakley. A lower bound for domination numbers of the queen's graph. J. Combin. Math. Combin. Comput., 43:231-254, 2002.

[25] W.D. Weakley. Upper bounds for domination numbers of the queen's graph. Discrete Math., 242:229-243, 2002.

[26] W.D. Weakley. Queens around the world in twenty-five years. In Graph Theory Favorite Conjectures and Open Problems, Volume 2, R. Gera, T.W. Haynes, S.T. Hedetniemi (Editors), Problem Books in Math., 43-54 (Springer, Cham, 2018). 